\title{
Genetics and Managed Care: Policy Statement of the American College of Medical Genetics
}

\author{
Marc S. Williams, MD, FAAP, FACMG
}

Hardly a day goes by without another announcement of some breakthrough relating to genetics or the Human Genome Project. Despite the potential for benefit that these advances represent, executives and medical directors of managed care organizations (MCOs) are appropriately concerned about the impact on patient care and the financial bottom line. Very few MCOs have integrated genetic services into day-to-day practice, whether in patient care, medical management, or coverage policy development.

\section{Brief history}

It is likely that the 20th century will be remembered in medical history largely as the century of human genetics. The rediscovery of Mendel's laws occurred in 1903. It was soon recognized that these laws apply to inheritance in humans, and the first genetic disorders-inborn errors of metabolism-were described in 1908. The structure of DNA was discovered in 1953, and within several years the mechanisms whereby genetic information is "read out" within the cell were coming into focus. The development of techniques to study human chromosomes led to the description of the first human chromosomal abnormality in 1959. The 1960s saw the advent of newborn screening programs for inborn errors of metabolism such as PKU and also the introduction of methods for prenatal diagnosis. In the 1970s, chromosome banding techniques became standardized and molecular methods were developed that made possible the isolation and purification of individual genes and the determination of gene sequences. Gradually, this spawned powerful new methods of molecular diagnosis and permitted elucidation of the pathophysiological mechanisms of many genetic disorders. The possibility of sequencing the human genome was envisioned in the 1980s, and, in 1991, the Human Genome Project was officially launched. It is fitting that a saga that began in the opening years of the 20th century culminated in the rough draft sequence of the entire complement of human genes in the last year of that century.

For most of this time, the medical applications of genetics were focused on rare disorders: inborn errors of metabolism, chromosomal abnormalities, and single gene conditions such as cystic fibrosis or sickle cell anemia. The overwhelming importance of genetic mechanisms in these disorders made them readily accessible for study. Although important advances in diagnosis and treatment have helped innumerable individuals

Associate Medical Director, Gundersen Lutheran Health Plan, and Chair, Committee on Economics of Genetic Services, American College of Medical Genetics. and families, genetics has had relatively little impact on the management of more common conditions that affect the vast majority of the population. With much of the human genome sequence in hand, we are poised to uncover the contribution of genes to more prevalent disorders such as diabetes, cancer, cardiovascular, neurodegenerative, and psychiatric disease, to name but a few. Already, this has led to the development of predictive tests for some disorders, together with the promise of new methods of diagnosis, prevention, and treatment. The practice of medicine is undergoing a transformation that will leave virtually no area untouched. Although there is legitimate concern that this new knowledge may cause harm if not applied thoughtfully, there is no question of the tremendous opportunity for widespread benefit.

A widespread misconception is that genetic conditions are rare and do not involve very many people. Although individual disorders may be rare, the aggregate burden of genetic disease is significant. (For example, it is estimated that $30 \%$ to $50 \%$ of admissions to pediatric inpatient facilities involve children with a genetic disorder.) Because of this perception, genetic disease is significantly under-recognized, which means that opportunities for prevention and anticipatory guidance are missed, with attendant costs to the patients, the families and the health care system in general. This aspect will be discussed in more detail below

\section{The specialty of medical genetics}

The growth of knowledge in human genetics has been paralleled by the development of the profession of medical genetics, which began to define itself in the 1960s. Training programs for clinical geneticists and counselors appeared in the 1970s. The American Board of Medical Genetics (ABMG) was established in 1982 and, 2 years later, administered its first certifying examinations for MDs, PhDs, and genetic counselors. The ABMG is one of 24 independent Boards that are fully approved by the American Board of Medical Specialties (ABMS). The ABMG, which was recognized in 1991, was the first independent Board recognized by the ABMS since 1979. In 1993, the American Board of Genetic Counseling was founded and assumed responsibility for certification of counselors.

The American College of Medical Genetics (ACMG) was incorporated in 1991 to provide a voice and a forum for certified clinical geneticists. In 1995, the ACMG became the newest member of the Council of Medical Specialty Societies. In 1996, the American Medical Association granted the College a seat in the House of Delegates and, in 2000, voted to create a Section 
on Genetics. There are now more than 3,000 individuals in the United States certified as medical geneticists. The specialty is unique in that it includes physicians, PhDs (who can be certified as medical geneticists or laboratory geneticists), and Masters degree genetic counselors. All have passed both a general genetics examination and one or more subtests in a specific area, such as counseling, cytogenetics, molecular genetics, etc. Recertification is mandatory for individuals certified in 1993 or later, and must be completed by passing the complete examination every 10 years or by participating in continuing education and passing a shorter examination every 2 years.

\section{Clinical geneticists}

Clinical geneticists are physicians who complete at least 2 years of residency training in a classical specialty, such as pediatrics, internal medicine, or obstetrics and gynecology, and then complete an additional 2 to 3 years of residency fellowship training in medical genetics. (Genetics residency programs are accredited by the American Council of Graduate Medical Education.) Clinical geneticists are concerned with the diagnosis and management of individuals with genetic disease. The best known example of the practice of clinical geneticist is that of a pediatric geneticist (the dysmorphologist) evaluating children with birth defects, mental retardation, or other rare disorders. Although this is a part of the practice, the stereotype minimizes the actual and potential range of services provided by clinical geneticists. They are involved in a wide variety of clinical endeavors, such as:

- Prenatal diagnostic evaluation and procedures.

- Evaluation of recurrent pregnancy loss and infertility.

- Evaluation of children and adults with mental retardation, birth defects, congenital malformations, chromosomal abnormalities, neurologic and neuromuscular problems.

- Analysis of single gene disorders, including disorders such as hemochromatosis, cystic fibrosis, sickle cell anemia, and the thrombophilias.

- Evaluation of genetic traits involved in common diseases, such as diabetes and heart disease.

\section{Biochemical geneticists}

A medical genetics subspecialist, the biochemical geneticist, has completed additional fellowship training, passed the ABMG subspecialty certification examination, and is an expert on the diagnosis and treatment of metabolic disease (so-called inborn errors of metabolism). Many of these diseases (such as PKU, galactosemia, and MCAD) are identified by state newborn screening programs. Biochemical geneticists include physicians who provide direct patient care and MDs or $\mathrm{PhDs}$ who direct or supervise laboratories that provide diagnostic testing and provide consultation to physicians. The expansion of newborn screening programs made possible by new technology will increase the need for biochemical geneticists to provide appropriate diagnosis and long-term management of these infants. Unfortunately, the number of clinical geneticists entering this subspecialty has been declining for several years.

\section{Cytogeneticists}

Cytogeneticists are MD or PhD level practitioners who have completed fellowship training in laboratory methods of chromosome preparation and analysis. Chromosome analysis is used in a variety of clinical settings, including, but not limited to:

- Prenatal diagnosis.

- Assessment of multiple miscarriage and stillbirths.

- Mental retardation.

- Multiple congenital anomalies.

- Infertility.

- Oncology.

In addition, the training these individuals receive in tissue culture and cell preservation frequently leads to development of tissue and DNA banking services under their supervision. Approximately $95 \%$ of the cytogenetics laboratories in the United States are directed by ABMG-certified cytogeneticists.

\section{Molecular geneticists}

Molecular genetics involves the analysis of genetic material to identify gene mutations and variants that are associated with genetic diseases or cancer. Tests are performed for disorders such as cystic fibrosis, muscular dystrophy, sickle cell anemia, breast cancer, colon cancer, hemochromatosis, and a rapidly expanding list of other disorders. Clinical molecular geneticists are MD or PhD level practitioners who have completed fellowship training in the area of molecular genetic analysis and have passed the ABMG examination in this specialty. These individuals will increasingly be called upon to develop new diagnostic tests based on knowledge of the molecular basis of disease and to assist physicians in the interpretation of these tests. They will be expected to translate the "raw data" of the Genome Project into clinical applications such as detection of disease susceptibility, pharmacogenetic analysis, and genetic risk profiling.

\section{PhD medical geneticists}

In addition to physician medical geneticists, PhDs may pursue training in medical genetics in an accredited program and take the appropriate certifying examination offered by the ABMG. PhD medical geneticists are experts in:

- Genetic risk assessment.

- Interpretation of genetic tests.

- Communication of this information to patients, family members, and other health providers.

Most work in academic institutions or are associated with genetic testing laboratories.

\section{Genetic counselors}

Genetic counseling is the process of explaining medical and scientific information about an inherited condition or birth defect to an individual or family. The goal of genetic counsel- 
ing is for families and individuals to understand the information, participate in decision-making about their medical care, and be able to manage the associated problems in a way that is best for them and their families. Genetic counselors are health care professionals who have completed training in an accredited Masters degree program and have passed the certification examination administered by the American Board of Genetic Counseling. Genetic Counselors are trained to:

- Review family histories and medical records.

- Discuss genetic conditions and how they are inherited.

- Explain inheritance patterns.

- Perform genetic risk assessments.

- Review available testing options.

- Discuss disease management, treatment and surveillance options.

- Explore the impact of genetic disorders on both affected and unaffected family members and assist families and individuals as they adjust to the diagnosis.

Most genetic counselors work in conjunction with a medical geneticist or as part of a department, program, or institution. They play a crucial role in health care delivery, particularly in the areas of prenatal diagnosis and cancer genetics.

\section{Genetics in managed care}

Medical genetics is a relatively new specialty and raises important questions for the MCO. How necessary are the services? Are they cost-effective? What is the cost of a "genetics benefit"? What laboratory tests are indicated and under what circumstances? These are just a few questions that arise. Although the need for such services is apparent to specialists in genetics, it is easy for MCOs to dismiss their assertions as selfserving. The problem is compounded by the fact that most medical schools offer only an introductory genetics course during the first or second year. This lack of training leaves most doctors woefully undereducated in a field that is expanding so rapidly that even its practitioners are hard-pressed to keep up. Consequently, many medical directors do not understand the role of geneticists in the health care delivery system. This lack of understanding frequently leads to marginalization of the clinical geneticist as one interested only in "rare disease" and the assumption that genetic services are high cost/low return or can be performed by the primary care physician according to published guidelines. Geneticists may at times contribute to this misunderstanding by narrowing service delivery to a small group of patients and being unresponsive to requests for help in other areas of genetics. Access is very limited under typical "gatekeeper" systems. Not only must primary care physicians recognize the value of genetic intervention to write a referral, but the medical management department of the MCO must agree and authorize the referral. Data regarding the clinical outcomes of genetic interventions and their cost-effectiveness are only beginning to emerge. In an effort to overcome these limitations, the ensuing discussion will identify situations in which partnerships between geneticists and MCOs may be mutually beneficial.

\section{Patient care}

The core tenet of clinical genetics is that establishing a specific and accurate diagnosis confers significant benefit to the patient and family. This seems counterintuitive given that cure or even treatment of most genetic disorders is currently beyond our abilities. Nonetheless, there are some benefits that can be identified, although their contribution to cost and patient satisfaction has yet to be quantified.

\section{Elimination of unnecessary tests}

Prader-Willi syndrome (PWS) offers a vivid example of an inexpensive test that can prevent distress to patients and their families and realize considerable savings for MCOs. In infancy, the patients are extremely hypotonic and have difficulty feeding, resulting in poor weight gain. As they mature, they present with mental retardation, short stature, and obesity with uncontrolled appetite, and are frequently worked up for neuromuscular and metabolic disorders by means of a myriad of laboratory tests, including muscle biopsy, with a price tag in the thousands of dollars. PWS is caused by abnormalities of chromosome 15, and the molecular diagnostic test can be done for approximately $\$ 200$ to $\$ 300$. Clearly, if it were done first, considerable savings would be realized and the patient would not have undergone a painful procedure that would have proved nondiagnostic.

\section{Information about recurrence risk to be used in reproductive decision-making}

When a child has serious medical problems, a key question for most parents is, "Will it happen again?" Unfortunately, all too frequently the response to this question is, "Don't worry. This is very rare, a one in a million occurrence." Failure to recognize a genetic disorder may result in an unanticipated recurrence that is potentially avoidable. MCOs may not be liable for these types of misadventures, but they are burdened with the medical costs that accrue from a second affected child with significant medical expenses. Assessment by a geneticist or genetic counselor can, in many cases, provide accurate information about recurrence that the parents can use in their reproductive decision-making. When couples elect not to have more children, or to use prenatal diagnostic tests to assist pregnancy management, MCOs are spared the expense of covering services for a second affected child. Even if parents choose not to use these options, savings can be realized in that anticipation of another affected child can influence management, avoiding costly complications. An example is a couple who had a child with a severe, untreatable metabolic disorder in which infants are initially normal but then develop problems that invariably result in death within days if supportive measures are not provided. Their first child was hospitalized in a neonatal intensive care unit for 3 months before the diagnosis was made and support was withdrawn. After being informed that there was a $25 \%$ risk of recurrence, the couple decided to have another pregnancy and declined prenatal diagnosis. At birth a diagnostic test was performed that indicated that this infant was also 
affected. The parents declined medical intervention and the baby died in the arms of this family at less than a week of age. Although the point of this approach was to provide the care the family desired, it also saved hundreds of thousands of dollars in health care expenditures when compared with futile measures taken for the first child. Even without a specific diagnosis, geneticists are frequently able to give accurate recurrence risks based on empirical data.

\section{Development of specific therapies for treatable conditions that result in improved health}

This is the primary rationale for newborn screening for disorders such as congenital hypothyroidism. Diagnosis in a newborn allows treatment with thyroid hormone, preventing the health problems and mental retardation associated with the disease. Although not all illnesses are treatable in this manner, there are many therapies that can improve health, such as immunoglobulin replacement in certain immunodeficiency syndromes, enzyme replacement, vitamin supplementation, and dietary modification.

\section{Provision of prognostic information that can be used to anticipate other problems seen in the condition and to develop condition-specific treatment plans}

A good example of this is Down syndrome, a condition with many associated health issues. Congenital heart disease is present in $40 \%$ of affected individuals. Echocardiography is indicated to identify significant disease and initiate treatment before onset of symptoms. Lifetime incidence of hypothyroidism is $25 \%$. Onset can be anytime, so annual thyroid testing is recommended to identify disease in the presymptomatic stages. This testing is particularly important because the symptom pattern for hypothyroidism significantly overlaps that of Down syndrome itself, making a clinical diagnosis extremely difficult. These examples and others have led to publication of recommendations for the care of children with Down syndrome by the American Academy of Pediatrics. This type of information is available for many common genetic conditions, but only a few of the rare ones. Guidelines may allow for primary care physicians to appropriately manage some genetic conditions but, given the rarity of most, clinical geneticists will need to be involved in the care of these patients.

\section{Technology assessment}

No other field of medicine is undergoing the rapidity of change that is seen in genetics. This finding puts significant pressure on MCOs regarding policy development and benefit enhancement. Geneticists can assist MCOs in determining the standard of care for genetic practice. An example of this relates to genetic testing. The "gold standard" for coverage of laboratory tests is FDA approval, and many benefit packages specifically exclude those tests not approved by the FDA. The majority of genetic tests present a problem, as the FDA has so far declined to evaluate them. A study of many genetic tests routinely covered by most MCOs (such as chromosome analysis) would reveal that many of them do not carry the FDA impri- matur. In its ongoing effort to address the issue of quality control and assessment, in 1999, the American College of Medical Genetics issued the second edition of its Standards and Guidelines for Clinical Genetics Laboratories ( http://www.faseb.org/genetics/acmg/stds/stdsmenu.htm). Recommendations of the Secretary's Advisory Committee on Genetic Testing (SACGT) may also help to clarify the issue of technologic assessment.

The characterization of genes that are responsible for inherited disorders has provided powerful tools for diagnosis based on detection of genetic mutations in samples of DNA. The proper use of these tests raises many complex issues in medical decision-making. In some cases, a test may indicate the potential for an individual to eventually develop a disorder, but whether the disorder will ever occur, or when, may be uncertain. An example is BRCA testing for breast cancer. A woman who is found to have a mutation has a $60 \%$ to $80 \%$ risk of developing breast cancer, an appreciable risk but not a certainty. Interpretation of results can also be challenging. Some tests do not detect all possible mutations, so an affected individual may, in some cases, test negative. Conversely, some genetic changes that are identified by testing are not associated with disease. Studies have shown, for example, that physicians commonly misinterpret the results of testing for colon cancer, giving either false reassurance or inappropriately high estimations of risk. Genetic tests must, therefore, be used and interpreted carefully, by physicians who understand the complexities. A medical geneticist can help to avoid the unnecessary use of expensive tests and can ensure that patients are accurately informed of the significance of test results. This can lead to savings for the MCO, as there would be assurance that the right test was being done on the right patient for the right reasons. Subsequent medical interventions that may depend on test results can also be managed appropriately.

\section{Utilization and medical management}

Appropriate diagnosis and counseling before genetic testing is essential for optimal medical management. Involving geneticists also allows for collection of utilization data. At present, such information is not available from claims data because the CPT and ICD-9 code sets are not sufficiently specific to cover the majority of genetic tests. Consequently, most are labeled with generic genetic test codes, which lack disease specificity. Geneticists can work with plan actuaries to develop epidemiological data that allow cost estimates to be conducted before discussion of benefit enhancement. They can also be looked upon as resources for in-service education of medical review nurses and medical directors. The ACMG is addressing the inadequate coding systems through the CPT Panel of the American Medical Association.

Another important consideration is the role of the clinical geneticist in the management of patients with complex genetic conditions. Primary care physicians may not have the experience, training, or time to adequately order and interpret the results of complex genetic tests, as is apparent from several studies. Encouraging referrals to clinical geneticists and using them as care coordinators for these patients should result in 
increased quality of care and the potential for decreased costs. In this way, clinical geneticists can provide a service similar to that of disease management programs for diabetes or congestive heart failure. Geneticists can assist with educational efforts for provider systems to facilitate implementation of new genetic knowledge in clinical practice. This will become increasingly important as the role of genetics in the management of common diseases expands.

The pharmacy benefit is a significant cost driver for most MCOs. Some of these dollars are spent on medications that prove to be ineffective for some patients. There are also costs associated with adverse reactions to medications. Although only a few examples currently exist, the field of pharmacogenomics has the potential to identify individual genetic variation that can predict which medications will be most effective for specific patients. It may also identify individuals at risk for adverse reactions. One can predict the emergence of a new specialty, pharmaceutical genetics, that will help MCOs and pharmacy benefit managers maximize the desired effects of medication and minimize the adverse reactions. This will improve quality of care and address some of the issues relating to the Institute of Medicine's report on medical errors.

\section{Quality of care}

Much of the foregoing discussion relates to quality of care. This section will focus on two additional matters relating to quality: access and quality improvement initiatives.

One aspect of access relates to the patient's need for access to high quality genetic information, the value of which has been discussed above. Another relates to the efficient use of the provider's time in dispensing this information. Consider, for example, a counseling session before amniocentesis. In addition to the elements necessary to informed consent, the session will also cover the indications for the test, the potential test results, and the patient's response to this information. These can be relatively straightforward if the indication is advanced maternal age, but they become increasingly complex if the indication is suspected fetal problems. Consequently, the time involved can range from 45 to 90 minutes. In cancer counseling, given the importance of compiling an accurate family history and the complexity of explaining the testing options, it is not unusual to spend more than 2 hours to allow adequate exchange of information. It is unrealistic to expect physicians to spend that much time with a patient or family. Genetic counselors can serve as "physician extenders," freeing the physician's time without compromising quality.

The managed care industry is one of the most tightly regulated in this country. Many of the regulations relate to quality of care, and women's health and maternal and child care are among the areas of greatest concern. Genetics can be used to build quality programs in these areas. Examples include familial breast and ovarian cancer programs in women's health and prenatal diagnosis programs in maternal and child health. Another area that seems likely to garner attention in MCOs is the care of special needs children. Clinical geneticists are already trained in disorder-specific management and could logically head such programs. As more knowledge emerges from the Human Genome Project, there may even be a role for the geneticist in such common disease management programs as diabetes and depression, particularly with respect to pharmaceutical intervention.

\section{Challenges}

Several challenges must be addressed before genetics is fully integrated into medical practice. Two have already been discussed: deficiencies in the CPT and ICD code sets and the potential pitfalls of heavy reliance on existing clinical guidelines. The reluctance of physicians to refer to geneticists has also been touched on, but there are other issues that impact this area. Despite the explosion of genetic knowledge in the past 10 years, there has been no expansion of time devoted to genetics in the curriculum of medical students in this country. Additionally, there are few opportunities, outside of electives, that allow medical students to experience the role of the geneticist or genetic counselor in care delivery. The situation in residencies is even worse, as, with very few exceptions, there is no formal involvement of genetics in resident education, especially in residencies that focus on adults. This affects not only the rate at which new genetic information is incorporated into clinical practice and the use of genetics professionals, but also the numbers of physicians who choose a career in genetics. The concern is that as more and more health care organizations wish to incorporate genetics into regular practice, training programs may be unable to accommodate the demand. Although the economics of physician supply and demand will usually adjust to provide sufficient providers, there may well be a lag of several years before the demand is met.

Reimbursement for the services of genetic counselors is limited. At present, counselors are licensed only in California and Utah. Without licensure, there are significant barriers to recognition of counselors as a billable entity. As a consequence, they are not reimbursed for their services by most third party payers. This has led to understandable reluctance on the part of medical systems to absorb the cost of these services when there is no opportunity to offset them. As the economic environment becomes increasingly stringent, these important services are increasingly at risk. Although potential solutions are beyond the scope of this discussion, MCOs have the ability to provide reimbursement to provider systems outside of a standard fee-for-service or capitation arrangement that can allow these services to be offered to their members.

The implementation of the privacy regulations of the Health Insurance Portability and Accountability Act (HIPAA) are a major concern of MCOs at this time. Although there are no "genetic specific" provisions of HIPAA, genetic practice has raised significant concerns regarding privacy and discrimination. Geneticists can assist MCOs in the development of policies and procedures that protect genetic information and prevent discrimination in accordance with recommendations of the Ethical, Legal, and Social Implications (ELSI) program of the National Human Genome Research Institute (NHGRI). 
The final question is of paramount importance to MCOs in the current competitive environment, namely, "What will it cost?" There are currently no published data that specifically address this issue. There are some large systems that offer a full range of genetics services (such as Northern California Kaiser Permanente) and are still able to remain competitive in their market, which suggests either that all of the competition is offering these services (which does not seem to be the case) or that the effect on the premium is not sufficient to decrease competitiveness.

\section{What can be done now?}

As genetics alters the practice of medicine, MCOs will have to adapt to these new approaches. What immediate actions should be considered by MCOs? The following suggestions seem reasonable and prudent:

1. Inventory the genetic services that are currently covered.

2. Assess the network for availability of and access to genetic providers.

3. Have genetic expertise available to the technology assessment team.
4. Assess the plan's ability to track case management and utilization of genetic tests.

5. Define and develop the role of genetic professionals in the network.

6. Develop policies and procedures that promote privacy of genetic information and prevent genetic discrimination.

7. Partner with other organizations to lobby for genetic counseling licensure at the individual state level.

8. Support and participate in dialogue between MCOs and genetics organizations to address the issues identified above.

In summary, this document provides an introduction to the role of the medical geneticist in the managed care environment. It is hoped that this information will encourage increased dialogue between geneticists and MCO medical directors with the ultimate goal of expanding access to genetics services and better serving our patients.

Approved by ACMG Board of Directors on July 1, 2001. (C) AMERICAN COLLEGE OF MEDICAL GENETICS, 2001

This guideline is designed primarily as an educational resource for medical geneticists and other health care providers to help them provide quality medical genetic services. Adherence to this policy statement does not necessarily assure a successful medical outcome. This policy statement should not be considered inclusive of all proper procedures and tests or exclusive of other procedures and tests that are reasonably directed to obtaining the same results. In determining the propriety of any specific procedure or test, the geneticist should apply his or her own professional judgment to the specific clinical circumstances presented by the individual patient or specimen. It may be prudent, however, to document in the patient's record the rationale for any significant deviation from this guideline. 